\title{
LA MATERIA PRIMERA \\ Y LA FORMA CORPOREITATIS \\ EN LA FÍSICA DE ALBERTO MAGNO
}

\author{
JIMENA PAZ LIMA \\ CONICET \\ Universidad Católica Argentina, Buenos Aires
}

\begin{abstract}
RESUMEN. La física de Alberto Magno se centra sobre la base de una materialidad que no puede ser definida como pura potencialidad pues no podría explicarse la continuidad y teleología propias de todo cambio sustancial. Por esto, en la materia primera existen ciertas dimensiones cuantitativas indeterminadas que la hacen divisible y le permiten sustentar a la forma sustancial en el cambio. Estas dimensiones suponen la presencia de una primera formalidad en la materia, llamada forma corporeitatis, que hacen de la materia primera una realidad actualizada e independiente de la forma sustancial.
\end{abstract}

PALABRAS CLAVE: Alberto Magno, física; materia primera, dimensiones indeterminatae, forma corporeitatis.

\section{Prime matter and forma corporeitatis in Albert the Great's physics}

ABSTRACT. Albert the Great's physics is based on a matter that cannot be defined as pure potentiality as it wouldn't be possible to explain the continuity and teleology of every substantial change. This is why, there are some indeterminate quantitative dimensions that make matter divisible and allow it to support substantial form in the natural motion. These dimensions represent the presence of a first form in matter, which is called forma corporeitatis and which makes matter become an actual reality and a reality that does not depend on substantial form.

KEY WORDS: Alberto Magno, física, materia primera, dimensiones indeterminatae, forma corporeitatis,.

\section{INTRODUCCIÓN}

La física o filosofía natural de Alberto Magno se centra en la afirmación de que la naturaleza es autosuficiente y autónoma en tanto que regula desde sí misma los diversos fenómenos y movimientos, prescindiendo así de la necesidad de un concurso divino para explicar el orden cósmico. En efecto, totalmente correcta es la naturaleza en sus operaciones ${ }^{1}$. Desde este paradigma, el pensador escolástico desarrolla una teoría de la materia y el cambio, observando que en la naturaleza todo cambio físico se produce continua y teleológicamente. Entendemos por cambio continuo a aquel movimiento que se produce ininterrumpidamente y sin mediación de reposo, y cambio teleológico a aquel que se ejecuta en dirección a un fin, lo que implica que no es azaroso o casual. Luego de la corrupción de cierta sustancia, sostiene Alberto, se genera siempre una misma sustancia; al clásico axioma quod nihil fit ex nihilo, el maestro dominico agrega, quod non quodlibet fit ex quolibet: una cosa

1 A. Magno, De mineralibus, 1.1 .5 (Borgnet vol. V: 8). «Certissima est natura in operationibus suis». 
no se genera a partir de cualquier cosa². Así, el autor intenta explicar por qué la materia primera de un compuesto recibe cierta forma sustancial y no otra en el proceso de cambio, es decir, por qué razón todo cambio sustancial se produce continua y teleológicamente.

Según el maestro dominico, el movimiento (motus) puede ser entendido de cuatro maneras diferentes: como actio, como passio, como fluxus formae identificado con el término final del movimiento (fluxus a fine in quo stat) y como fluxus formae diferente del fin del movimiento (fluxus per essentiam [...] differt ab eo a quo fluit). De estas cuatro posibilidades, Alberto descarta que el movimiento sea definido como mera acción o pasión, afirmando que es un fluxus formae esencialmente uno con el término final del movimiento, y que igualmente es un fluxus formae esencialmente diferente del fin del movimiento. El maestro dominico afirma que el movimiento debe entenderse como un ininterrumpido fluxus formae y señala lo complejo que es observar el cambio en su transición y diferentes etapas, en tanto es más simple observar un cuerpo ya modificado ${ }^{3}$. Por otra parte, es en la observación del resultado del cambio — según el maestro dominico- en donde se hace evidente la causalidad con la que se produce cualquier generación y corrupción.

Alberto Magno intenta dar una explicación cabal para la cuestión de la materia y el movimiento, pues considera que la teoría hilemórfica de Aristóteles es insuficiente para dar razones de los procesos de generación y corrupción de un ente. En efecto, afirmar que la materia es pura potencialidad no justifica que reciba determinada forma en el cambio en lugar de cualquier otra. Según el filósofo, desde una teoría de la materia primera en tanto mera indeterminación

2 A. Magno, Metaphysica, 11. 1. 8 (Cologne t. XVI: 470.64-65). También ver De caelo, 1. 4. 10 (Cologne t. V-1: 103. 5-12). «Quod naturalia non sunt a casu nec a voluntate, sed a causa agente et terminante ea, nec nos in naturalibus habemus inquirere, qualiter deus opifex secundum suam liberimam voluntatem creatis ab ipso utatur ad miraculum, quo declaret potentiam suam, sed potius quid in rebus naturalibus secundum causas naturae insitas naturaliter fieri possit».

Además, sugeirmos consultar S. SNYDER, Albert the Great, Incohatio Formae, pp. 63-82; y A. Rodolfi, Il concetto di materia nell'opera di Alberto Magno, p. 119.

3 Cf. A. Magno, Physica, 3. 1. 8 (Cologne t. IV: 163-168). También ver B. Ashley, St. Albert and the Nature of Natural Science, p. 84; y E. McCullough, St. Albert on Motion as Forma fluens and Fluxus formae, pp. 129-153.

Es interesante destacar que, según Anneliese Maier, Alberto Magno procura integrar la noción de movimiento como fluxus formae (tomada de Avicena) con el movimiento como forma fluens (tomado de Averroes), tesis que comparte William Wallace. Sin embargo, según Ernest McCullough, una lectura seria al De motu ofrece poca evidencia que corrobore tal intento de integración y sostiene que, en el estudio de Maier, existen múltiples «dificultades conceptuales». McCullough, en definitiva, afirma que el movimiento en la doctrina albertina es entendido únicamente como constante fluir de la forma, es decir, como constante fluxus formae, tesis que Alberto efectivamente toma de la obra aviceniana Sufficientia.

Respecto a la bibliografía, además del estudio de McCullough que ya hemos citado, sugerimos consultar: A. MaIER, Die Vorlaufer Galileis im 14. Jahrhundert, pp. 9-25; W. WaLLace, Causality and Scientific Explanation, pp. 67-69; y O. Lizzini, Fluxus. Some remarks on Avicena and Albertus Magnus, pp. 1-13. 
no es posible explicar por qué razón todo cambio sustancial necesariamente se produce de manera ininterrumpida y en vistas hacia un fin en particular.

Por esta razón, afirma Alberto, es menester que exista en la materia primera algo que justifique la acción de recibir cierta forma y no otra en el proceso de cambio; debe existir algo en la materia que evidencie la naturalidad con la que se produce toda generación. Así, la materia se encuentra compuesta por ciertas formas que la disponen a ser informada por determinada forma sustancial y no por otra en la futura generación del compuesto. Estas formas que se encuentran presentes en la materia primera, previamente al advenimiento de la forma sustancial, son denominadas por Alberto Magno: forma corporeitatis e incohatio formae 4 .

\section{LOS DISTINTOS NOMBRES Y DEFINICIONES DE LA MATERIA}

Tal como figura en De quatuor coaequaevis, Alberto Magno presenta cuatro definiciones distintas de materia, tres de ellas dadas por los físicos que estudian a la materia en tanto existente en la realidad, y una de ellas a cargo de los metafísicos que abordan el tema considerando a la materia como principio fundante del ente corpóreo:

«en la primera parte del De Generatione et Corruptione, se define a la materia como hyle en tanto es propia y esencialmente sujeto de la generación y corrupción, y en tanto es susceptible de ser transmutada en otras cosas. También, en Metaphysica VIII, dije que la materia no es esto en acto, sino que es en potencia. Además, en Metaphysica IX: la materia es esto según lo que se ve porque aquello que es según el contacto, y no según el orden, es la materia. Asimismo, al final de la primera parte de la Physica, dije que la materia es el sujeto primero desde lo cual algo se hace» ${ }^{5}$.

Como vemos, en la primera definición, la materia es considerada como sujeto de la generación y corrupción; en la segunda, como ente en potencia para la forma; en la tercera, como aquello que puede ser tocado, es decir que se la identifica con un cuerpo y con un ente que puede ser conocido por los sentidos. La cuarta definición, en cambio, a cargo de los metafísicos, asume a la materia como principio constitutivo del ente.

Por otra parte, Alberto utiliza distintos nombres para referirse a la materia:

4 En particular, se desarrollará en este trabajo la noción de forma corporeitatis, dejando la incohatio formae para otras investigaciones.

5 A. Magno, De quatuor coaequaevis, 1. 2. 2 (Borgnet vol. XXXIV: 323). «In primo de Generatione et Corruptione, diffinitur sic materia: est autem hyle maxime quidem et proprie subiecum generationis et corruptionis et susceptibile, modo autem alio est quod aliis transmutationibus. Item, in VIII Metaphysicae: dico materiam illud quod non est hoc in actu, sed est hoc in potentia. Item, in XI Metaphysicae: materia est hoc secundum quod videtur: quoniam illud quod est secundum contactum, et non est secundum ordinem, est materia. Item, in fine primi Physicorum: dico materiam primum subiectum unicuique, ex quo fit aliquid». También ver A. Rodolfi, Il concetto di materia nell'opera di Alberto Magno, pp. 23-33. 
hyle en tanto se encuentra en potencia de recibir una forma; subiectum por su capacidad de sustentar a la forma sustancial y subiectum primum por soportar a las formas accidentales; materia en tanto constituye un principio del compuesto; massa, como sinónimo de «materia prima» y por cuanto puede ser modificada innumerables veces por causa de las diferentes formas que puede recibir; origo, en tanto es primera en la composición de una sustancia y en tanto origina al movimiento; y elementum puesto que, si bien desde la composición de una sustancia es primera, desde el análisis de su descomposición, es última ${ }^{6}$.

Asimismo, en diversas oportunidades Alberto se opone a distintas posturas que, desde su concepción, confunden a la materia con otra cosa. En primer lugar, por ejemplo, en De quatuor coaequaevis ${ }^{7}$ y en la Summa theologiae ${ }^{8}$, el filósofo rechaza categóricamente el panteísmo materialista esgrimido por David de Dinant, que considera que la única realidad es la materia, que Dios y la materia son la misma cosa y que ésta última es increada ${ }^{9}$.

En segundo lugar, en In II Sententiarum ${ }^{10}$, el maestro dominico se opone al hilemorfismo universal de Avicebrón, quien aplica la composición aristotélica de materia y forma a la totalidad de los entes. En efecto, según Avicebrón, no sólo las sustancias materiales se encuentran compuestas por materia y forma, sino que también los entes espirituales están constituidos por forma y materia espiritual. Respecto a esto, así como Alberto Magno rechaza la

6 Cf. A. MAgno, Physica, 1. 3. 12 (Cologne t. IV: 60.61-61.21). "Hoc autem principium, quod est materia, multa habet nomina, quorum quaedam sunt propria et quaedam per translationem conveniunt ei. Nomina autem, quibus nominatur proprie sunt hyle, subiectum, massa, materia, origo et elementum. Nomina autem, quibus nominatur transumptive, sunt silva, mater, femina. Nominum autem propriorum Avicenna in Sufficientia reddit rationem talem dicens, quod dicitur hyle, secundum quod ipsa est receptibilis formarum in potentia aut formae. Subiectum autem dicitur secundum hoc, quod est in actu sustinens formam aut motum ad formam (...) gratia ipsius substare habet omne quod substat, propter quod etiam ipsa vocatur subiectum primum et substantia composita prima vel secunda dicitur subiectum secundum. Materia autem est, secundum quod omnia resolvuntur in illam sicut un alterum componentium; massa vero, secundum quod ipsa una prima existens per divisionem formarum est in omnibus compositis substantiis diversificata, sicut vasa figuli ex una massa luti finguntur diversificata formis artificialibus. Origo autem dicitur, inquantum ad ipsa rei incipit compositio vel inquantum ab ipsa est motus. Sic autem est immixta privationi, ut diximus, quia per privationem, quae permixta est ei, principium efficitur, et rei origo, a qua, si intelligeretur abstracta, posset quidem esse in composito alterum componentium cum forma, non tamen subici posset motui propter causas, quas supra assignavimus (...). Elementum autem dicitur, secundum quod est in resolutione ultimum et in compositione primum. Et in ipsa stat resolutio, quia ipsa non resolvitur in aliquid aliud aliquod modo resolutionis, quia elementum simplicior est pars compositi et uno modo simplicior est quam forma, quoniam forma speciei, quae est in individuo hoc signato, contingit resolvere in diffinentia sicut docetur in septimo philosophiae primae».

Para este tema, ver A. Rodolfi, Il concetto di materia nell'opera di Alberto Magno, pp. 18-19.

7 Cf. A. Magno, De quatuor coaequaevis, 1. 5. 2 (Borgnet vol. XXXIV: 69).

8 Cf. A. Magno, Summa theologiae, I. tr. 4. q 20. c. 2 (Cologne t. XXXIV: 104.25-47).

9 Para la posición albertina en torno al pensamiento de David de Dinant, cf. A. CAPARello, Alberto Magno contra David di Dinant, pp. 156-180; y A. Rodolfi, pp. 60-64.

10 Cf. A. Magno, In II Sententiarum, d.1. a.4 (Borgnet vol. XXVII: 13-15). 
posibilidad de que Dios, el intelecto y la materia sean una misma cosa, tesis de Dinant, tampoco acepta la existencia de una materia espiritual, como defiende Avicebrón. Según Alberto ${ }^{11}$, sólo de un modo impropio el fundamentum de la sustancia angélica puede ser llamado materia espiritual pues, en rigor, sólo existe un único tipo de materia: la materia de las sustancias que se generan y corrompen. El maestro dominico sostiene que las sustancias inmateriales no están compuestas por forma y materia espiritual, sino por un suppositum y por la forma de ese suppositum ${ }^{12}$.

\section{LA FORMA CORPOREITATIS Y LAS DIMENSIONES INDETERMINATAE DE LA MATERIA}

En primer lugar, Alberto Magno sostiene que la materia prima de los cuerpos terrestres no puede ser entendida como pura potencialidad pues, en ese caso, sería en sí misma de carácter indivisible. El cambio, por el contrario, sólo puede producirse en un sujeto capaz de división en partes. Asimismo, si se definiera a la materia como pura potencialidad, ¿cómo explicar que sea capaz de recibir y sustentar a la forma sustancial en la generación del compuesto? Es necesario, pues, que exista en la materia prima una forma que la haga divisible y sujeta a dimensiones cuantitativas; una forma que le otorgue a la materia cierta cantidad y que le permita actuar como auténtico receptáculo de la forma sustancial en el cambio. Esta forma, denominada por Alberto Magno, forma corporeitatis, es la primera formalidad de la materia primera que pre-existe en ella antes de que ésta sea informada por la forma sustancial.

Así,

«como la materia, siendo completamente indivisible, no puede ser dividida en partes cuantitativas (...), es necesario que, en orden a recibir una forma que sea acto del cuerpo, la materia primero tenga que convertirse en capaz de recibir tres dimensiones. La materia tiene que ser capaz de recibir dimensiones, pero no tiene que tener dimensiones determinadas porque si las tuviera, se corrompería en cada generación y corrupción. Por esta razón, la materia recibe primero una forma, que es la forma de dimensiones indeterminadas, a través de la cual la materia se vuelve divisible en partes y es capaz de recibir la forma sustancial. Esta corporeidad es una forma que es común a todas las formas que recibe la materia. Entonces, la corporeidad de la materia, la cual es como una primera forma antes de todas las otras formas que son perfecciones del cuerpo, permanece en la materia y nunca es separada de ella en ningún cambio corporal, y si se despojara de esta forma, sucedería que el cuerpo devendría desde un no cuerpo absolutamente y devendría en cuerpo de la nada absolutamente, lo cual mostramos que es imposible, según la naturaleza,

11 Cf. A. Magno, In II Sententiarum, d.3. a 4 (Borgnet vol. XXVII: 66-69).

12 Cf. A. Magno, De quatuor coaequaevis, 1. 2. 5. (Borgnet vol XXXIV: 334).

Para la posición albertina en torno a la filosofía de Avicebrón, cf. J. Weisheipl, Albertus Magnus and Universal Hylemorphism: Avicebron, pp. 239-260; y A. Rodolfi, Il concetto di materia nell'opera di Alberto Magno, pp. 64-71. 
en el primer libro de la Física (...). Esta corporeidad es la primera forma, por la cual [la materia] recibe a todas las formas sustanciales del cuerpo» ${ }^{13}$.

Además, la forma corporeitatis de la sustancia nunca abandona a la materia, y está en ella misma [en la materia] antes de todo cambio y mutación ${ }^{14}$.

La forma corporeitatis o, como también la denomina Alberto, corporeitas, es pues una cierta formalidad que le corresponde a la materia prima antes de que ésta sea determinada por la forma sustancial. Esta forma de la corporeidad, siendo una forma physica o forma materiae, le otorga a la materia primera ciertas dimensiones, a la vez que le permite ser divisible en partes. Ambas cosas aseguran que, en el futuro cambio sustancial, la materia pueda comportarse efectivamente como receptáculo o soporte de una determinada forma sustancial, y que asimismo pueda ser dividida en partes. Si la materia no poseyera primero ciertas dimensiones y no fuera capaz de dividirse, jamás podría producirse el cambio sustancial. Por esto, en acto, la forma corporeitatis le da a la materia cierta cantidad (dimensiones), al mismo tiempo que le da la potencia para continuar modificando su cantidad, la potencia para continuar dividiéndose. Y esto, es decir, la división en acto y la

13 A. MAGno, De caelo, 1. 3. 4 (Cologne t. V-1: 62.59-63; 63.7-8). "Cum autem materia non dividatur ita, quod per partes subiaciatur diversis formis nisi per quantitatem, eo quod secundum se indivisibilis est, sicut ostendimus in primo Physicorum, oportet, quod materia, quae suscipit formas, quae sunt actus et perfectiones corporis, sit primo susceptibilis trium dimensionum, non tamen terminatarum ad certam dimensionem, quia si certae dimensiones essent materiae, contingeret, quod continue corrumperentur in omni generatione et corruptione; et hoc non esse verum ostendimus in primo Physicorum. Propter quod suscipit primo formam, quae est corporeitas indeterminatarum dimensionum, qua mediante divisibilis efficitur et diversis formis substantialibus per partes subicibilis Et haec corporeitas est communis omnibus formis, quas suscipit, quia, sicut ostendi habet in secundo Peri geneseos, forma communis in materia omnibus vel pluribus substantiis non mutatur, quando mutantur formae substantiales. Sicut patet, quando mutatur ignis in aerem, remanent caliditas et diaphanitas eaedem in essentia, licit habeant diversum esse in igne et in aere, et ita corporeitas materiae, quae est sicut forma prima respectu omnium formarum, quae sunt perfectiones corporeae, remanet in ipsa, et numquam dedudatur ab ipsa in aliqua transmutatione quorumcumque corporum; et si denudaretur, contingeret, quod corpus esset ex omnino non-corpore et quod fieret corpus ex nihilo omnino, et hoc ostendimus impossibile esse secundum naturam in primo Physicorum (...). Quia igitur haec corporeitas prima forma est, per quam suscipit omnem formam substantialem corporis».

También, por ejemplo, ver: Physica 1. 2.5 (Cologne t. IV: 24.16-18). «Contingit ideo, quia non ingreditur materiata sua nisi sub prima forma sua, quae est corporeitas, et per illam efficitur divisibilis».

Además, se puede consultar A. Rodolfi, Il concetto di materia nell'opera di Alberto Magno, pp. 98-99; y S. BALDnER, Sources of St. Thomas' Teaching on Prime Matter or Albert and Thomas on Matter.

14 Alberto Magno, Metaphysica, 5. 3. 2 (Cologne t. XVI: 259.37-40). "Quod forma corporeitatis substantialis non relinquit umquam materiam et est in ipsa ante omnem motum et mutationem»; y Metaphysica, 5. 2. 4, (Cologne t. XVI: 239. 68-71): «ex omnibus autem his manifestum est materiam a corporeitate nullo modo posse separari. Quod autem materia sit maioris vel minoris spatti vel rara vel densa vel figurae huius vel illius, hoc habet a forma physica». 
divisibilidad en potencia de la materia primera, es lo que en efecto posibilita el futuro cambio sustancial.

Además, como se comprende a partir del texto recién citado, la forma de la corporeidad no le otorga a la materia dimensiones determinadas (dimensiones terminatarum) porque si esto fuera así, la materia conformaría un compuesto que se corrompería en cada generación. La corporeidad, pues, le otorga a la materia primera dimensiones de tipo indeterminadas (corporeitas indeterminatarum dimensionum o corporeitas non terminatarum dimensionum), las cuales son determinadas luego por la forma sustancial del compuesto.

Por otra parte, la forma de la corporeidad es una forma que es común al resto de las formas que recibe una misma materia, lo que significa que esta forma guarda una cierta relación con la forma sustancial del futuro compuesto. Todas estas formas se relacionan entre sí y, juntas, cooperan en la generación del compuesto. La «corporeidad» permanece en el cuerpo, sin perderse en ningún tipo de cambio por los que la materia tuviera que atravesar. Por consiguiente, se podría suponer que la forma corporeitatis no sólo pre-existe en la materia antes de cualquier cambio, sino que además subsiste en la materia segunda del compuesto luego de la generación.

En torno al tipo de formalidad que conviene a la corporeitas, Alberto Magno sostiene que ésta no se trata de una forma sustancial o de una forma accidental, sino más bien de una forma intermedia entre una y otra. El maestro dominico postula la existencia de ciertas cuasi-formas sustanciales o formas intermedias en orden a poder explicar la generación y corrupción. Así, el orden para la forma (...) es un intermedio entre el ente y el no ente ${ }^{15}$. La cosmología albertina, en efecto, admite un tercer tipo de formalidad ${ }^{16}$. Más aún, esta forma materiae o forma physica es llamada por el maestro dominico, forma partis, para distinguirla de la forma sustancial del compuesto, denominada forma totius. De esta manera,

«las sustancias que se generan y corrompen están conformadas por materia y forma. Pero del compuesto no se predica la forma de la materia (forma materiae o forma partis) sino la forma total del compuesto (forma totius coniuncti)» ${ }^{17}$.

La primera formalidad presente en la materia prima, pues, recibe el nombre de forma partis en tanto informa únicamente a la materia, y por ello se dice

15 Alberto Magno, De quatuor coaequaevis, 1. 2. 4 (Borgnet vol. XXXIV: 329-330). «Ordo ad formam (...) medium est inter ens et non ens». También ver L. DewaN, St. Albert, Creation, and the Philosophers, p. 297; S. BALDnER, Sources of St. Thomas' Teaching on Prime Matter or Albert and Thomas on Matter; y S. BALDNER, St. Albert the Great: the matter of the heavens, pp. 7-8.

16 Cf. S. Baldner, Sources of St. Thomas' Teaching on Prime Matter or Albert and Thomas on Matter; S. BALDner, St. Albert the Great: the matter of the heavens, pp. 5-8.

17 A. MAGno, De quatuor coaequaevis, 1. 2. 5 (Borgnet vol. XXXIV: 334). «In quibusdam enim substantiis est compositio ex materia et forma, sicut in generabilibus et corruptibilibus, quorum neutrum praedicatur de substantia composita: compositum enim neque materia est, neque forma, unde in talibus, universale quod praedicatur de composito, non accipitur a forma materiae, sed a forma totius coniuncti». También ver A. Rodolfi, Il concetto di materia nell'opera di Alberto Magno, p. 69. 
también que es forma materiae. Esta forma es determinada con la llegada de la forma totius coniuncti al compuesto. La forma sustancial de la materia segunda es llamada forma totius por cuanto informa y modifica a la sustancia en su totalidad. No obstante, esta distinción de formas conviene únicamente a las sustancias materiales, ya sean animadas o no, pues las sustancias espirituales no poseen composición de materia y forma, sino suppositum y forma, tal como advierte Alberto Magno contra Avicebrón ${ }^{18}$.

Ahora bien, se entiende que la forma de la corporeidad modifica a la materia primera y segunda de los cuerpos terrestres, sin embargo cabe preguntarse qué ocurre en el caso de los cuerpos celestes. La cosmología medieval, heredera de la aristotélica, considera que la materia celeste es distinta de la materia terrestre. Una prueba de esto, afirma el maestro dominico, es que los cuerpos celestes no pueden transmutar en cuerpos terrestres, ni viceversa ${ }^{19}$. La materia del mundo lunar corresponde al quinto elemento denominado éter y no posee mezcla de los cuatro elementos básicos y primitivos, propios de los cuerpos sublunares. Por esto, mientras los cuerpos del mundo terrestre sufren todo tipo de cambios, los cuerpos celestes sólo se mueven localmente, lo que significa que no cambian cuantitativa ni cualitativamente, ni se generan o corrompen ${ }^{20}$. Los astros y los entes conformados por el quinto elemento poseen un movimiento circular eterno, un movimiento perpetuo, perfecto y regular ${ }^{21}$.

De esta manera, Alberto Magno señala que la forma de la corporeidad corresponde únicamente a la materia de los cuerpos terrestres. En efecto, dado que los cuerpos celestes no padecen cambios sustanciales, la materia de estos cuerpos no necesita primero recibir ciertas dimensiones indeterminadas por medio de la forma de la corporeidad para luego poder recibir a la forma sustancial en la generación del compuesto pues, en rigor, estos entes no se generan ni corrompen. Además, según la astronomía clásica, las inteligencias puras o formas celestes se encuentran totalmente separadas de sus cuerpos, no necesitando de la forma corporeitatis para que actúe como mediadora entre la materia y la forma. Así, los cuerpos celestes (...) no reciben a las formas sustanciales a través de la mediación de la corporeidad [es decir, a través de la forma corporeitatis]22.

18 Cf. A. MAgno, De quatuor coaequaevis, 1. 2. 5 (Borgnet vol. XXXIV: 334). También A. Rodolfi, Il concetto di materia nell'opera di Alberto Magno, p. 69.

19 Cf., A. Magno, De quatuor coaequaevis, 1. 2. 5 (Borgnet vol. XXXIV: 336). "Sed superiorum corpum non transmutatio ad invicem ergo ipsorum non est materia una». También ver A. Rodolfi, La dottrina della materia celeste in Alberto Magno, p. 32.

20 Cf. S. Baldner, St. Albert the Great: the matter of the heavens, pp. 1-4; A. CRombie, Historia de la Ciencia: De San Agustín a Galileo, pp. 76-94; A. Rodolfi, La dottrina della materia celeste in Alberto Magno, pp. 25-47; S. Baldner, Sources of St. Thomas' Teaching on Prime Matter or Albert and Thomas on Matter; y B. PRICE, The Physical Astronomy and Astrology of Albertus Magnus, pp. 155-185.

21 Cf. A. Magno, De caelo, 1. 3. 4 (Cologne t. V-1: 61-65). También ver S. Baldner, Sources of St. Thomas' Teaching on Prime Matter or Albert and Thomas on Matter; S. BALDNER, St. Albert the Great: the matter of the heavens, pp. 1-4; y A. Rodolfi, La dottrina della materia celeste in Alberto Magno, pp. 26-27.

22 A. Magno, De caelo, 1. 3. 4 (Cologne t. V-1: 63.63-64). «Caelum (...), cum tamen substantiales formas suas primas, mediante corporeitate non recipiant». 
Las formas puras no son formas de los cuerpos celestes, más bien son sus motores, de modo que estos cuerpos no se comportan como auténticos receptáculos de las formas. No obstante, el maestro dominico sostiene que verdaderamente los astros se encuentran compuestos por materia y forma, discutiendo con Averroes quien, en De substantia orbis, afirma que sólo equívocamente es posible referirse a su composición hilemórfica ${ }^{23}$.

Los cuerpos celestes, entonces, carecen de la corporeitas en tanto, por un lado, no padecen cambios sustanciales y, por el otro, se encuentran separados de las inteligencias o formas puras. La falta de esta primera formalidad en la materia celeste es la causante, a su vez, de la inmutabilidad sustancial del mundo lunar. Si, pues, la forma corporeitatis permite a la materia terrestre ser divisible y ser sujeto de dimensiones cuantitativas, la falta de dicha formalidad provoca la indivisibilidad del mundo de los astros. Y, entonces, si la materia de los cuerpos celestes no es susceptible de ser dividida en partes, no puede corromperse, pues la destrucción implica la separación en partes. De modo que los cuerpos celestes, a diferencia de los terrestres, no son divisibles y no se generan ni corrompen ${ }^{24}$. Por esto, según Steven Baldner,

«Alberto admite dos tipos de materia primera: se encuentra la materia prima de los cielos, que carece de una forma corporeitatis inherente, y la materia prima de la tierra, que posee esta forma coporeitatis. La materia prima es, por supuesto, absolutamente carente de forma y, sin embargo, cierta materia prima posee una corporeidad inherente y otra no» ${ }^{25}$.

Finalmente, en De caelo 1.3.4, Alberto Magno sostiene que las formas celestes se distinguen de las formas de la corporeidad en tanto las primeras son creadas por Dios desde la nada y las segundas, en cambio, son producidas por la propia potencialidad de la materia. De esta manera,

«Aristóteles determinó que estas formas [las formas de los cuerpos celestes] no sean traídas al ser por un sujeto material, como [sí ocurre con] las formas de los cuerpos [con la forma corporeitatis que] son traídas al ser por la potencia de la materia. Más bien, todas esas formas son traídas al ser por la Causa Primera (...). Por lo tanto, Aristóteles dijo que las sustancias separadas

23 Cf. A. Magno, De quatuor coaequaevis, 3. 7. 2 (Borgnet vol. XXXIV: 403). También ver A. Rodolfi, La dottrina della materia celeste in Alberto Magno, pp. 27-33.

${ }^{24}$ Cf. A. Magno, De caelo, 1. 3. 4 (Cologne t. V-1: 61-65). También ver S. BaLdner, Sources of St. Thomas' Teaching on Prime Matter or Albert and Thomas on Matter; S. BALDNER, St. Albert the Great: on form and matter, pp. 6-7; S. BALDNER, St. Albert the Great: the matter of the heavens, pp. 4-8; y A. Rodolfi, La dottrina della materia celeste in Alberto Magno, pp. 28-47.

25 S. BALDNER, Sources of St. Thomas' Teaching on Prime Matter or Albert and Thomas on Matter. "In effect, then, Albert has presented us with a doctrine of two types of prime matter; there is the prime matter of the heavens, which lacks an inherent forma corporeitatis, and the prime matter of the earth, which has an inherent forma corporeitatis. Prime matter is, of course, absolutely without form, and yet some prime matter has an inherent corporeity and some does not». 
y el intelecto humano tienen que haber sido traídos al ser por una causa extrínseca [y no por la potencia de la materia, como la forma corporeitatis] ${ }^{26}$.

Sin embargo, en una obra coetánea al comentario al texto de Aristóteles, en Super Dionysium De div. nominibus cap. 4, el maestro dominico sostiene: si buscamos de dónde viene la primera forma en la materia [la forma corporeitatis], decimos, según Avicena, que viene por creación ${ }^{27}$.

No queda en claro, entonces, si la primera forma de la materia es causada por la propia corporalidad o si, por el contrario, es creada por Dios. Se cree que los comentarios a las obras de Aristóteles fueron elaborados durante los mismos años en los que el maestro dominico trabaja sobre los comentarios a las obras del pseudo-Dionisio. De modo que no hay registro de que Alberto haya sostenido primero una tesis y luego la haya refutado; más bien encontramos dos tesis contrarias en obras coetáneas. Pensamos que el uso de los conceptos responde a necesidades hermenéuticas diferentes, puesto que se enmarcan dentro de la lectura de filosofías heterogéneas, como son las del estagirita y la de Dionisio.

En suma, la materia primera de los cuerpos terrestres posee en sí misma ciertas dimensiones indeterminatae que le permiten ser sujeto del cambio

26 A. Magno, De caelo, 1. 3. 4 (Cologne t. V-1: 63.63-64). «Et illa [sententia] quidem quae est Aristotelis, sicut conici potest ex verbis suis, licet in nullo loco expresse dicat in libris, quorum notitia ad nos pervenit, est, quod intelligentia sit forma caeli et quod caeli materia sit susceptibilis huiusmodi formae ante corporeitatem. Propter quod Aristoteles frequenter dicit, quod non est una materia caeli et corporum generabilium et corruptibilium. Et quod caelum habet corporeitatem determinatarum dimensionum et rotundarum superficierum, hoc non est ideo, quod istae dimensiones eductae sint de potentia materiae, quae potentia est dimensionis indeterminatae, qua recipit formas corporis, sed potius, quia motus caeli non est nisi corporis. Et quia intelligentiae sunt moventes, oportuit caelum habere determinatas dimensiones, cum tamen substantiales formas suas primas mediante corporeitate non recipiant. Et haec est causa, quare substantiae intellectuales sunt impartibiles et immobiles per se, quia non movetur nisi corpus et non dividitur nisi quantum vel quod est in quanto sicut in subiecto. Et quia sunt indivisibiles divisione subiecti, ideo determinavit Aristoteles, quod tales formae sunt non constitutae per subiectum sive per materiam, quemadmodum corporum formae omnes per materiam consituuntur quoad hoc quod educuntur de materia sicut actus de potentia. Sed potius omnes istae sunt constitutae a causa prima secundum omnes ordines caelorum et caelestium corporum. Et ideo dixit Aristoteles eas esse substantias separatas et hac eadem de causa dixit intellectum hominis ingredi ab extrinseco, quia omnis forma educta de potentia ad actum secundum potestatem materiae educitur et non potest esse nisi limitatae operationis, quia cum materia efficitur actu, tunc dividitur et non dividitur nisi per corporeitatem, ut diximus, et ideo omnis forma educta de materia sicut de potentia, est consequens corporeitatem. Dico autem consequens natura, non tempore».

También ver Metaphysica 11. 1. 8 (Cologne t. XVI: 470.44-45). «Omnis forma educitur de potentia».

Respecto a la bibliografía, sugerimos consultar S. BALDner, Sources of St. Thomas Teaching on Prime Matter or Albert and Thomas on Matter; S. BALDNER, St. Albert the Great: on form and matter, pp. 6-7; y S. BALDNER, St. Albert the Great: the matter of the heavens, pp. 5-8.

27 A. Magno, Super Dionysium De div. nominibus, c. 4 (Cologne t. XXXVII-1: 285). «Et si quaeratur, unde veniat prima forma in materia, dicendum secundum Avicennam, quod per creationem». 
sustancial en tanto posibilitan su divisibilidad en partes, conditio sine qua non para el cambio. Además, sin estas dimensiones, la materia primera no podría recibir y sustentar a la forma sustancial en la generación del compuesto. Como se explicó, la cantidad de la materia primera es de tipo indeterminada en tanto es delimitada en pesos y tamaños específicos con el advenimiento de la forma sustancial al compuesto. Esta cantidad implica la presencia de una primera formalidad en la materia primera, llamada forma corporeitatis, la cual es una forma intermedia entre la sustancial y la accidental. La corporeitas es considerada forma materiae y en este sentido forma partis, diferente de la forma totius que actualiza a la totalidad del compuesto. Por último, la forma de la corporeidad corresponde únicamente a la materia primera del mundo sublunar por cuanto la materia celeste es incorruptible e indivisible.

De esta manera, Alberto Magno procura explicar la continuidad y teleología con la que se desarrolla todo cambio sustancial en tanto non quodlibet fit ex quolibet. En la medida en que la materia primera posea cantidad y no sea pura potencia e indeterminación, se encontrará preparada y dispuesta para recibir cierta forma sustancial y no cualquier otra en la generación del compuesto, garantizando así la teleología del cambio. Asimismo, como se explicó oportunamente, la forma corporeitatis no abandona a la materia en ningún cambio, lo que implica que no sólo dispone a la materia primera sino también a la materia segunda del compuesto. En este sentido, según Alberto, se garantiza la continuidad del cambio en tanto es producido sin mediación alguna de reposo. La teoría hilemórfica de Aristóteles, según el pensamiento albertino, no justifica estas dos propiedades del cambio sustancial, la continuidad y teleología, por cuanto la materia entendida como pura potencialidad se encuentra abierta a recibir infinitas formas, lo que implicaría que el cambio se produciría azarosa y casualmente.

\section{LA MATERIA PRIMERA COMO REALIDAD ACTUALIZADA}

La cosmología de Alberto Magno plantea la inexistencia de la materia primera como pura potencialidad en sentido aristotélico. En efecto, el maestro dominico hace una distinción entre el término potentia, que remite a la materia como pura indeterminación y se vincula al pensamiento del estagirita; y el término potestas, que refiere a la doctrina de la formalidad pre-existente en la materia primera y a la concepción de la materia como entidad actualizada ${ }^{28}$. Según el maestro dominico, la materia no puede ser concebida como mera potentia ad esse, sino más bien como potestas stans per actus incohationem pues, de lo contrario, no podrían explicarse las propiedades fundamentales de la generación y corrupción.

Por esto, la forma de la corporeidad implica en la materia primera un real aptitudo ad actum, que convierte la mera indeterminación de la «materia primera» en la determinación de la «materia propia», condición necesaria para

28 Cf. O. Lizzini, Fluxus. Some remarks on Avicena and Albertus Magnus, pp. 10-12. 
el natural desarrollo del cambio sustancial ${ }^{29}$. La materia no es entendida en la obra albertina como pura potencialidad y principio indeterminado, más bien se la concibe como una entidad compuesta por cantidad y por una formalidad virtual, tesis que se encuentra basada en el ideal de la materia como cierta actualidad o cierta potencialidad activa.

Además, en Physica 1.3.13, Alberto Magno se pregunta si la materia primera es completamente simple, mientras la consideremos en sí misma y sin encontrarse informada por la forma sustancial. Frente a esto, el maestro dominico responde: ergo ipsa simplex non est. La materia prima no es una realidad en sí misma simple pues justamente — antes del cambio-, se encuentra informada por cierta cantidad y por la forma de la corporeidad, sin la cual no sería posible explicar el cambio sustancial. La materia se encuentra compuesta por el quod est y por la relatio hacia la forma sustancial, es decir, por el quod est y por la forma corporeitatis que implica una real composición en la materia primera, una cierta actualidad o formalidad. La corporeitas constituye en la materia una tendencia cuasi-formal hacia la forma sustancial, o incluso la presencia de una quaedam res formalis en la materia primera. Esta última, en definitiva, no es pura potencialidad ni absolutamente simple ${ }^{30}$.

Ahora bien, se podría suponer que el maestro de colonia introduce una real composición en la materia en orden a distinguirla de la absoluta simplicidad de Dios. En efecto, se dice que Dios es verdaderamente simple porque todas las otras cosas son, de algún modo, compuestas ${ }^{31}$. No obstante, mientras en Summa

29 Cf. B. NARDI, Studi di filosofia medievale, pp. 85-93 y A. Rodolfi, Il concetto di materia nell'opera di Alberto Magno, p. 110.

30 Cf. A. Magno, Physica, 1. 3. 13 (Cologne t. IV: 62.81-64.18). "In contrarium autem huius est, quod talis materia aut potest esse subiectum formae aut non. Si non potest esse subiectum formae, ergo impossibile est ipsam umquam formae subici, quod falsum est, sum nos videamus eam esse subiectum. Si autem potest, aliqua potentia potest. Sed non est idem id quod potest, et potentia eius; ergo ipsa simplex non est».

También, De quatuor coaequaevis, 1. 2. 4 (Borgnet vol. XXXIV: 329-330). «Utrum materia sit simplex vel composita? Simplex (...) non habens compositionem penitus (...) nisi tantum ordinis ad formam cum substantia materiae: et hic ordo differt ab ipsa, sicut potentia (...) ita scilicet quod non habet compositionem in aliquo quod est res simpliciter; (...) ordo ad formam... medium est inter ens et non ens».

Además, ver Physica, 1. 3. 10 (Cologne t. IV: 56.58-66) y Metaphysica, 11. 1.7 (Cologne t. XVI: 467.80-468.7)

Para este tema, consultar S. BALDNER, St. Albert the Great: the principles of nature, pp. 1-10; S. Baldner, St. Albert the Great: on form and matter, pp. 1-9; L. Dewan, St. Albert, Creation, and the Philosophers, pp. 295-307 y A. Rodolfi, Il concetto di materia nell'opera di Alberto Magno, pp. 50-60.

31 A. MAGNo, In I Sententiarum, d. 8. a. 24. sol. a q. (Borgnet vol. XXXV: 254). «Dicitur Deus vere simplex: quia omnia alia aliquo modo sunt composita: proprie autem quia hoc soli sibi convenit».

También ver Physica, 1. 3. 13 (Cologne t. IV: 63.26-29). «Hic autem modus simplicitatis non convenit nisi primae causae; illa enim est omne id quod habet in se, quia illa est sua voluntas et sua potentia et sua intelligentia».

Respecto de la bibliografía, consultar S. BALDNER, St. Albert the Great: the principles of nature, p. 10 y A. Rodolfi, Il concetto di materia nell'opera di Alberto Magno, pp. 54-60. 
theologiae ${ }^{32}$ Alberto considera a la simplicidad como atributo exclusivo de Dios, en De quatuor coaequaevis ${ }^{33}$ sostiene que también la materia es simple, aunque condicionadamente. En efecto, se puede hablar de simplicidad en dos modos diferentes: por un lado, la simplicidad de Dios gracias a la cual sus propiedades se identifican con su esencia, y asimismo no depende de ningún otro ser; por el otro, la simplicidad de la materia en tanto no se encuentra compuesta por ningún elemento o parte. Por tanto, se puede hablar de simplicidad, tanto para el caso de Dios como para el de la materia, aunque en este último sólo se puede hablar de una simplicidad condicionada, pues en rigor la materia es en sí misma compuesta.

Más aún, la composición de la materia está dada por una doble causa: por un lado, en la materia existe una compositio ad intra debido a la presencia de las dimensiones indeterminatae y de la forma corporeitatis previamente a la generación del compuesto; y, por el otro, una compositio ad extra debido a su dependencia exclusiva de Dios y en tanto es creada y no es eterna ${ }^{34}$. Respecto de la composición externa, agrega el maestro dominico, sirve de fundamento para dos de las propiedades fundamentales de la materia, a saber, la mutabilidad y temporalidad. Pues, al ser creada desde la nada, la materia comienza a ser post nihil, lo que significa que es temporal y no eterna. De la misma manera, todo lo que es creado tiene un movimiento propio que lo distingue de Dios, quien es la primera causa y absolutamente inmóvil ${ }^{35}$.

32 Cf. A. MAGNO, Summa theologiae, I. tr 4. q 20. c.3 (Cologne t.XXXIV: 104-107).

33 Cf. A. Magno, De quatuor coaequaevis, 1. 2. 4 (Borgnet vol. XXXIV: 330). «Materia quae est subiectum generationis et corruptionis, et principium hoc modo quo subiectum potest esse principium, simplex est, non habens compositionem penitus, sicut dicunt auctoriates Augustini et probant ratione inductae, nisi tantum ordinis ad formam cum substantia materiae». También ver A. Rodolfi, Il concetto di materia nell'opera di Alberto Magno, pp. 54-60.

34 Cf. A. Magno, Summa theologiae, II. tr. 1. m. 3. a 2 (Borgnet vol. XXXII: 35). «Dupliciter contingit loqui de simplicitate. Est enim simplicitas omnimoda, in qua nulla est plica diversitatis secundum esse aliquo modo: et haec non convenit nisi Deo, quia nulla plica habitudinis ad aliquid intra vel extra depende secundum esse diversum a seipso: et haec est simplex omnino et omnimodo in fine simplicitatis: quae simplicitas nulli creato convenire potest. Et est simplicitas quae non habet plicam habitudinis secundum esse ad componens intra, licet habeat dependentiam ad causam extra, cuius est dare esse, et facere debere esse in omnibus quae sunt: et haec simplicitas non est omnino simplex, sed concreta habitudinibus multis, secundum dependentias quas habet ad principia sui esse: et hoc modo simplex est materia, et simplex forma, et simplex quod est, et simplex quo est: et haec est simplicitas quae potest convenire creato: hoc enim quamvis non componatur aliquando ex partibus componentibus esse totius, tamen semper dependet secundum esse ad causam efficientem a qua accipit esse: et haec dependentia essentialiter non est ipsum, quamvis inseparabilis sit ab ipso: et ideo non omnino simplex».

También consultar A. Rodolfi, Il concetto di materia nell'opera di Alberto Magno, pp. 57-58.

35 Cf. A. Magno, Summa theologiae, II. tr. 1. m 3. a 2 (Borgnet vol. XXXII: 31). «Constat autem quod secumdum Augustinum mutabile sequitur compositum: dicit enim, quod omne compositum mutabile est (...) dicit enim Augustinus in libro Contra epistolam fundamenti, quod <res non est mutabilis quia est ex aliquo, sed quia est ex nihilo>». También ver A. Rodolfi, Il concetto di materia nell'opera di Alberto Magno, p. 58. 
Por otra parte, según Alberto,

«la materia no se limita a ser el sujeto [subiectum] del cambio y del movimiento, más bien es un ente fundante [fundans ens] e individuante [individuans] y también, de esta manera, una entidad subsistente [substans entitati]. Y son todas estas cosas las que vienen antes de que [ésta] sea sujeto del movimiento, porque solamente es sujeto del cambio y del movimiento en tanto que es fundada [fundatum], individual [individuum] y subsistente [substans]» ${ }^{36}$.

La materia, así, no participa meramente como sustrato del cambio sustancial o como aquello que subyace al cambio; más bien participa como ente fundante, es decir, como aquel que funda al compuesto; como ente individuante, esto es, como principio de individuación del compuesto; y como ente subsistente, es decir, como sustancia. De esta manera, además de fundar, individuar y constituir una sustancia del compuesto, en sí misma y antes del cambio sustancial, la materia es un ente fundado, un individuo y una sustancia. Y, de hecho, puesto que en sí misma la materia es un ente fundado, un individuo y una sustancia, es que luego puede comportarse como sujeto o sustrato del cambio sustancial.

Por todo esto, Alberto Magno afirma que la materia tiene ser [esse] de sujeto y de potencia, y [que] lo tiene por sí misma, no como esse simpliciter, sino en tanto secundum quid ${ }^{37}$.

Además, sostiene que

«la forma no le confiere ser [esse materiae] a la materia, porque la materia tiene ser [esse materiae] en sí misma, más bien la forma sólo confiere ser al compuesto» ${ }^{38}$.

Si bien, entonces, la materia no es una sustancia completamente (esse simpliciter), sino secundum quid o relativamente, más bien constituye una entidad existente de por sí e independiente de la forma, una entidad que posee un esse materiae en sí misma y que no requiere de la forma para existir. La materia

36 A. MaGno, Metaphysica, 3. 3. 1 (Cologne t. XVI: 139.77-94). «Licet enim causa unde motus et materia et finis videantur mobilis in eo quod mobile est, esse principia, tamen (...) est materia non determinata per subiectum mutationis et motus, sed potius per hoc quod ipsa est fundans ens et individuans et substans entitati et huiusmodi, quae sunt ante motus subiectum, eo quod non subicitur mutationi et motui nisi fundatum et individuum et substans, et non convertitur, quod omne fundatum et individuatum et substans in seipso mutationi subiciatur et motui (...). Et quoad huiusmodi considerationes (...) prima materia substans (...) determinare habet primus philosphus».

Ver, también, S. Baldner, St. Albert the Great: on form and matter, pp. 1-9 y L. Dewan, St. Albert, Creation, and the Philosophers, p. 303.

37 Alberto Magno, De quatuor coaequaevis, 1. 2.2 (Borgnet vol. XXXIV: 321): «materia autem habet esse subiecti et potentia; et hoc habet a seipsa, et hoc non est esse simpliciter, sed secundum quid».

38 A. Magno, Metaphysica, 1. 4. 9 (Cologne t. XVI: 60.24-41). «Licet autem forma nihil conferat materiae de esse materiae, eo quod esse materiae habet materia a seipsa, tamen composito sola confert esse forma». También ver 1. 4. 8 (Cologne t. XVI: 56. 90-49).

Respecto de la bibliografía, sugerimos consultar S. BALDNER, St. Albert the Great: on form and matter, pp. 1-9; L. Dewan, St. Albert, Creation, and the Philosophers, pp. 301-306 y A. Rodolfi, Il concetto di materia nell'opera di Alberto Magno, pp. 37-44. 
tiene un ser propio que no deriva de la forma; esta última no es causa formal del ser de la materia, sino causa formal del ser del compuesto. La materia primera no es pura potencia ni absolutamente simple, sino una realidad actualizada, compuesta por una formalidad previa a la forma sustancial del compuesto. La materia no es un co-principio que participa pasivamente del cambio sustancial, sino una realidad en sí misma compuesta y actualizada, portadora de un elemento formal. La esencia de la materia no es, pues, potencia, más bien la materia posee potencia pero no se reduce a ella. En efecto, en De quatuor coaequaevis 1.2.2, Physica 1.3.13 y Metaphysica 1.4.9, Alberto Magno asegura que la esencia de la materia es distinta de su potencia, y que esto corresponde a una distinción de razón. Así, la potencia (...) es distinta de la materia. Pero esto no lo digo por la cosa, sino por la razón ${ }^{39}$.

A diferencia de Tomás de Aquino, entonces, en la doctrina albertina la materia primera no se identifica con su potencia, más bien constituye una realidad actualizada, que posee potencia para la forma, pero que no es únicamente ello. La potencia no entra en la definición de materia, y entre materia y potencia no existe una distinción real sino de razón.

Ahora bien, no sólo sostiene el maestro dominico que la materia posee un ser independiente de la forma, sino que también la forma es independiente de la materia. Así,

«se dice forma a a lo que permanece afuera [quasi foris manens], y cuanto más afuera se encuentra de la materia, en la sustancia, en el ser y en la operación, más verdaderamente se denomina forma. Y así, el intelecto [el alma intelectual] es más verdaderamente forma que los sentidos [que el alma sensitiva]» ${ }^{40}$.

De modo que, así como la materia es independiente de la forma, así también la forma lo es de la materia. Y cuanto más la forma se encuentra separada de la materia, más verdaderamente es forma, y esto mismo ocurre con la materia. Por esto, según Alberto, el alma intelectual es más forma que el alma sensitiva, puesto que la primera se encuentra más separada del cuerpo que la segunda. Forma y materia, cuanto más separadas una de la otra, mayor poder y capacidad poseen; ambas son principios del ente, aunque no principios una de la otra ${ }^{41}$.

39 A. Magno, Physica, 1. 3. 13 (Cologne t. IV: 63.86-90). «Potentia, qua subiectum esse potest, diversa est a materia. Sed non dicit rem, sed rationem, qua refertur ad formam, et illi potentiae substat per seipsam, quia materia ex seipsa subiectum est formae primae vel potentiae ad formam primam». También ver A. Rodolfi, Il concetto di materia nell'opera di Alberto Magno, pp. 52-54.

40 A. Magno, Metaphysica, 1. 4. 9 (Cologne t. XVI: 60.27-31). «Forma enim est quasi foris manens dicta, et quanto plus manet foras materiam substantia et esse et operatione, verius habet nomen formae. Et ideo intellectus verius est forma quam sensus». Cfr. S. BALDNER, St. Albert the Great: on form and matter, p. 2 y L. Dewan, St. Albert, Creation, and the Philosophers, pp. 305-306.

41 Cf. A. Magno, Metaphysica, 5. 2. 4 (Cologne t. XVI: 239.93-98). «Materia enim non est causa substantiae formae, quia id quod est in potentia non est causa eius quod est in effectu (...), forma etiam non est causa quare materia sit substantia vel quare materia sit materia». También ver A. Rodolfi, Il concetto di materia nell'opera di Alberto Magno, p. 41. 
Forma y materia son dos realidades existentes de por sí e independientes una de la otra, que juntas intentan constituir un único ente.

\section{CONSIDERACIONES FINALES}

A modo de síntesis, según la doctrina albertina, una cosa no se genera a partir de cualquier cosa, por el contrario, la generación y corrupción son siempre continuas y teleológicas. Por tanto, previamente al cambio sustancial, es necesario que exista en la materia primera una forma intermedia denominada forma corporeitatis. Esta última constituye una cuasi-forma sustancial, es decir que no es una forma sustancial completamente ni tampoco accidental, la cual permite a la materia recibir y sustentar a la futura forma sustancial del compuesto. La forma de la corporeidad otorga a la materia cierta cantidad y dimensiones cuantitativas indeterminadas, las cuales luego del cambio son delimitadas por la forma sustancial. Esta forma no se separa nunca de la materia, en ningún cambio corporal, y sin ella no podría producirse ninguna generación y corrupción, pues hace a la materia divisible y dividida.

Respecto del origen de esta primera formalidad en la materia, Alberto no mantiene una posición única. Por un lado, afirma que es causada por la propia potencialidad de la materia; por el otro, que es originada a partir de la creación de Dios.

La forma corporeitatis pertenece únicamente a la materia primera de los cuerpos terrestres en tanto los cuerpos celestes, compuestos por el quinto elemento, no requieren de la forma de la corporeidad para hacer de mediadora entre la forma pura y la materia celeste. En efecto, las formas de los astros se encuentran separadas de los cuerpos y se comportan como «motor» y «móvil», razón por la cual los entes compuestos de éter no se generan ni corrompen.

Las dimensiones cuantitativas indeterminadas permiten a la materia primera poseer cierto tamaño y cantidad, gracias a lo cual la materia puede recibir y sustentar a la forma sustancial del futuro compuesto. De modo que estas dimensiones, aunque aún indeterminadas, sirven de fundamento para la continuidad y teleología que caracterizan a todo proceso de cambio físico.

En la formulación albertina, se considera a la materia primera como portadora de ciertas dimensiones y formalidades propias que no dependen de la forma sustancial. La materia primera, en la doctrina de Alberto Magno, no es considerada como pura potencia, sino más bien como potencialidad activa, como realidad existente de por sí e independiente de la forma sustancial. La materia primera posee un esse materiae propio, teniendo una existencia autónoma y separada de la forma sustancial del compuesto.

El maestro dominico considera insuficiente la teoría hilemórfica de Aristóteles para explicar los fenómenos de la naturaleza. Por esta razón, redefine las nociones de materia y forma, sosteniendo que la materia no es meramente potencia y afirmando que existen formas intermedias entre las sustanciales y las accidentales, tales como la forma de la corporeidad. La 
materia primera, en el planteo dualista de Alberto, se presenta como una realidad existente en sí misma, portadora de determinadas dimensiones cuantitativas y de cierta formalidad que no se subordina a la forma sustancial del compuesto. Alberto Magno reconsidera los principios metafísicos fundamentales del aristotelismo, exponiendo nuevos principios constitutivos de la realidad bajo una clara inspiración del pensamiento neo-platónico y árabe dominante en siglo XIII.

\section{BIBLIOGRAFÍA CITADA}

Alberti Magni, Opera Omina, Paris, Emil Borgnet (ed.), 1890-1899.

Alberti Magni, Opera Omnia, Münster, Aschendorff Verlag (ed. Cologne), 1951.

Ashley, Benedict M., O.P., St. "Albert and the Nature of Natural Science», en Weisheipl, James, A (comp.), Albertus Magnus and the Sciences. Commemorative Essays 1980, Toronto, The Hunter Rose co. Ltd, 1980, pp. 73-102.

Baldner, Steven, Sources of St. Thomas' Teaching on Prime Matter or Albert and Thomas on Matter: http://maritain.nd.edu/jmc/ti00/baldner.htm [consulta: 10/06/2010].

Baldner, Steven, St. Albert the Great: on form and matter, seminario dictado en University of Oxford: Undergraduate Course, Blackfriars Hall, Trinity Term 2006, pp. 1-9.

Baldner, Steven, St. Albert the Great: the matter of the heavens, seminario dictado en University of Oxford: Undergraduate Course, Blackfriars Hall, Trinity Term 2006, pp. 1-9.

Baldner, Steven, St. Albert the Great: the principles of nature, seminario dictado en University of Oxford: Undergraduate Course, Blackfriars Hall, Trinity Term 2006, pp. $1-10$.

Caparello, Adriana, «Alberto Magno contra David di Dinant: uno strano materialismo», Doctor Communis 48 (1995) pp. 156-180.

Crombie, Alistair, C., Historia de la Ciencia: De San Agustín a Galileo II-1, Madrid, Ed. Alianza, 1974.

Dewan, Lawrence, «St. Albert, Creation, and the Philosophers», Laval théologique et philosophique 40/3 (1984) pp. 295-307.

Lizzini, Olga, Fluxus. «Some remarks on Avicena and Albertus Magnus», ponencia presentada en "Workshop: Translation and Transformation in Philosophy: Albert, between Aquinas and 'the Arabs, Leuven, 5 june 2012, pp. 1-13.

Maier, Anneliese, Die Vorlaufer Galileis im 14. Jahrhundert, Roma, Edizioni de Storia e Letteratura, 1949.

McCullough, Ernest, J., «St. Albert on Motion as Forma fluens and Fluxus formae», en Weisheipl, James, A (comp.), Albertus Magnus and the Sciences. Commemorative Essays 1980, Toronto, The Hunter Rose co. Ltd, 1980, pp. 129-153.

Nardi, Bruno, Studi di filosofia medievale, Roma, Editioni di Storia e Letteratura, 1960.

Price, Betsy, B., «The Physical Astronomy and Astrology of Albertus Magnus», en Weisheipl, James, A. (comp.), Albertus Magnus and the Sciences. Commemorative Essays 1980, Toronto, The Hunter Rose co. Ltd, 1980, pp. 155-185.

Rodolfi, AnNa, "La dottrina della materia celeste in Alberto Magno», Medioevo e Rinascimento 17/14 (2003) pp. 25-47. 
Rodolfi, Anna, Il concetto di materia nell'opera di Alberto Magno, Firenze, Sismel. Edizioni del Galluzzo, 2004.

Snyder, Steven, «Albert the Great, Incohatio Formae, and the Pure Potentiality of Matter», American Catholic Philosophical Quarterly 70/1 (1996) pp. 63-82.

Wallace, William, Causality and Scientific Explanation 2 vols., Ann Arbor, University of Michigan Press, 1972.

WeisheIPL, JAmEs, A., «Albertus Magnus and Universal Hylemorphism: Avicebron. A Note on 13th-Century Augustinianism», en Kovach, Francis, J.; Shahan, Robert, W.; (ed.), Albert the Great: Commemorative Essays, Oklahoma, University of Oklahoma Press, 1980, pp. 239-260.

Facultad de Filosofía y Letras

Jimena Paz Lima

Universidad Católica Argentina, Buenos Aires

CONICET

jimelima@hotmail.com

[Artículo aprobado para publicación en diciembre de 2015] 